\title{
Use of Serial Weight and Length Measurements in Children from Birth to Two Years of Age to Predict Obesity at Five Years of Age
}

\author{
Harold S. Haller ${ }^{1 *}$, Tanujit Dey ${ }^{2}$, \\ Lisaann S. Gittner ${ }^{3}$ and Susan M. Ludington-Hoe ${ }^{4}$ \\ ${ }^{1}$ Case Western Reserve University, ${ }^{2}$ College of William \& Mary, ${ }^{3}$ College of \\ Public Service and Urban Affairs and ${ }^{4}$ Case Western Reserve University
}

\begin{abstract}
Childhood obesity is a major health concern. The associated health risks dramatically reduce lifespan and increase healthcare costs. The goal was to develop methodology to identify as early in life as possible whether or not a child would become obese at age five. This diagnostic tool would facilitate clinical monitoring to prevent and or minimize obesity. Obesity is measured by Body Mass Index (BMI), but an improved metric, the ratio of weight to height (or length) (WOH), is proposed from this research for detecting early obesity. Results of this research demonstrate that WOH performs better than BMI for early detection of obesity in individuals using a longitudinal decision analysis (LDA), which is essentially an individuals type control chart analysis about a trend line. Utilizing LDA, the odds of obesity of a child at age five is indicated before the second birthday with $95 \%$ sensitivity and $97 \%$ specificity. Further, obesity at age five is indicated with $75 \%$ specificity before two months and with $84 \%$ specificity before three months of age. These results warrant expanding this study to larger cohorts of normal, overweight, and obese children at age five from different healthcare facilities to test the applicability of this novel diagnostic tool.
\end{abstract}

Key words: Control chart analysis, decision analysis, infant obesity, LDA, regression.

\section{Introduction}

An increasingly common and growing public health problem currently in the United States is obesity. Though the obesity epidemic appears to have stabilized, the number of obese people continues to increase by the millions. The obesity

${ }^{*}$ Corresponding author. 
plague is costing the US billions of dollars and countless number of lives every year, burdening the health care system, straining economic resources, and having far reaching social consequences [1]. Hospital costs alone associated with childhood obesity were estimated at $\$ 127$ million during 1997-1999 (in 2001 constant U.S. dollars), up from $\$ 35$ million during 1979-1981 [2]. Obese children are prone to develop health disorders such as type-2 diabetes [3], high blood pressure [4, $5]$ and high cholesterol, which are risk factors for cardiovascular disease (CVD) $[6,7]$, cancer [8], infertility, dermatological disorders, breathing problems such as sleep apnea and asthma $[9,10]$, emotional disorder resulting in low self-esteem, depression and eating disorders [11], and stigma [12-14].

Although obesity is usually not measured in pre-school children, in one study comparing the year 2000 with the year 2003, obesity in a cohort of 2-5 year old children increased from $3.6 \%$ to $13.9 \%( \pm 1.6 \%)[19]$. In the year 2000 , Cole, Belllizzi, Flegal, and Dietz [15] noted that the United States had the highest prevalence of pediatric obesity when compared to 13 European countries. One might think that with all the efforts of recent US Administrations to emphasize the importance of proper eating and exercise among young children, the incidence of obesity in children under five years of age would be decreasing as parents have more control over the physical activities and eating habits of children of this age group. However, according to the Healthy People 2010 Midcourse Review performed in 2007, pediatric obesity increased and moved away from the target by $5 \%$ (United States DHHS, 2007). Since pediatric obesity is correlated with a number of serious medical complications and increased mortality in both children and adults as reported by Power, Lake, and Cole [16, 17], prevention of obesity at the earliest age without affecting normal growth is critical. In fact the US DHHS noted in 2007 that rather than treatment, prevention may hold the highest potential in reversing the trend in obesity in the US. Accurately identifying the tendency toward overweight and obesity in individuals by two years of age (yoa), may provide sufficient lead time for parents and pediatricians to take corrective action in the form of interventions related to eating habits and physical activities to reverse the tendency for children to be overweight and obese by the age of five. Such interventions may have the long term effect of transforming children's attitudes toward a healthier future lifestyle, though this is just a conjecture.

\section{Data}

Currently, obesity is frequently measured using Body Mass Index (BMI), an index for relating body weight to height, which is a person's weight in kilograms $(\mathrm{kg})$ divided by their height in meters $(\mathrm{m})$ squared. It has been shown that BMI data can be used to track average trends and discriminate between age-groups of the population over time, birth to age five, that are normal, overweight, and 
obese at age five based on World Health Organization (WHO) standards [18]. But measuring childhood obesity remains an arduous task, and there are likely potential biases that may affect the outcomes of studies in this area [18]. Few studies have used statistical methods to characterize the genesis of this epidemic for individuals at an early age. Thus, the inspiration for this study is to show that a method could be developed that would indicate as early as possibly before five years of age, whether or not an individual could be obese at age five so that an intervention could be recommended to the child's parent(s) or guardian(s) by a clinician, pediatrician, or family doctor. Thus a descriptive comparative longitudinal study of individuals using retrospective chart review from an earlier study [17] was planned. Data were extracted from records of pediatric "well child" visits from birth to five years and the maternal pregnancy record for the child-mother dyads. Children's records were classified into weight gender cohorts (normal, overweight, and obese) by their 5-year BMI based on WHO growth percentile classifications shown in Table 1. Data were obtained from a Midwestern HMO in Northeast Ohio serving approximately 150,000 people. All records of mothers and children born between 1997 and 2001 were reviewed. The data base contained records of "well-child" visits at 1-week; 2, 4, 6, 9, 12, 15, 18 months; and $2,3,4$, and 5 years. Mean days-since-birth (dsb) was used as individual child's age so the "well child" visit data could be compared.

Table 1: WHO guideline for detecting obesity at the age five

\begin{tabular}{lcc}
\hline WHO BMI Status at 5 yoa & Boys & Girls \\
\hline Healthy or Normal Weight & 14.5 to 16.6 & 14.3 to 16.9 \\
Overweight & 16.7 to 18.1 & 16.9 to 18.7 \\
Obese & $\geq 18.2$ & $\geq 18.8$ \\
\hline
\end{tabular}

Forty thousand $(40,000)$ mother-children dyad's records were reviewed by a computer generated algorithm based on an inclusion criteria; 800 records were randomly selected. Charts were manually reviewed by data collection teams, extracted with data recorded on a data collection tool and were verified and cleaned by a second team. Data collection teams were trained to attain a reliability of at least $0.8 \%$ agreement. Data were entered into SPSS 15.0 and again cleaned by selecting every third chart for data re-entry. Verification of data values was performed by referring back to the charts.

Inclusion criteria: at 5-years the child could be classified as either normal, overweight, or obese (using BMI, normal is the $25^{\text {th }}$ to $84^{\text {th }}$ percentile, overweight is the $85^{\text {th }}$ to $94^{\text {th }}$ percentile, obese is $\geq 95^{\text {th }}$ percentile as defined by WHO growth standards, see Table 1), had complete medical records for at least 8 of 12 wellchild visits from birth to 5 years old, was born at term after an uncomplicated gestation and had no medical complications other than non-food allergies, and 
the mother's pregnancy medical records were available.

Exclusion criteria: congenital defects (chromosomal defects, physical handicaps), premature birth (less than 38 weeks), a 5 minute APGAR Score of $\leq 7$, any trauma or medical complication that might affect growth, and maternal preexisting diabetes or gestational diabetes during the woman's pregnancy.

Records were manually reviewed for exclusion criteria, yielding 223 children that met both inclusion and exclusion criteria as shown in Table 2. However, for this study to maximize the contrast, only data from the normal and obese cohorts were used. Data collected from infants were: gender; APGAR Score; birth weight; weight at well-child visits; height from birth to 5 years; date of well-child visits; predominant feeding method at each visit from birth to six months; and timing of solid food introduction. Due to the retrospective nature of the study, height and weight measurements were assumed to be accurate and reliable to within 1-2\% as has been found in clinical practice. Days-since-birth (dsb), the actual age in days of the child on the date of the well-child visit, was used in the development of the growth pattern curves for each child using a longitudinal model that was parameterized similar to the models used to generate the WHO growth curves.

Table 2: Number of children in each category

\begin{tabular}{ll}
\hline Weight Status Based on BMI at 5 yoa & Number of Children \\
\hline Healthy & 61 \\
Overweight & 47 \\
Obese & 115 \\
\hline
\end{tabular}

As previously mentioned, a motivator for this study was to find a simple, accurate way to monitor an individual infant's growth-weight profile in order to detect those who could become overweight and obese at age five, which is the earliest age that the WHO categorizes children as being normal weight, overweight, or obese. By having a predictor of the tendency of an individual to become overweight or obese, a clinician, pediatrician or family physician could intervene with the parent(s) to change behaviors that might contribute to overweight and obesity before the age of five without any recommendations, such as feeding infants less and unfairly labeling them as fat, which was a concern of Dr. Michelle Lampl, director of Emory University's Center for the Study of Human Health (Lampl's comment was published in an article by Lindsey Tanner of the Associated Press entitled "Bigger Isn't Better" 11/8/11) [19].

Keys et al. [20] review several indices based on weight and height relative to anthropometry and statistics: weight divided by height (WOH), weight divided by height squared (BMI), weight divided by height cubed (Rohrer index), cube root of weight divided by height (Ponderal index), and the height divided by the cube root of weight (Shelton index). This study analyzed 7,424 healthy men in 
12 cohorts from five countries as well as 180 young men and 248 men who aged from 49 to 59. Keys et al. concluded that Ponderal index was the poorest of these relative weight height indices and that BMI was the best but only slightly better than WOH for the non-infant, male populations studied. Because BMI and $\mathrm{WOH}$ were only marginally different from Keys et al. study, the two metrics were considered: BMI and $\mathrm{WOH}$ for studying the relation between weight and height and obesity. The WOH metric for children under the age of five is much like the specific gravity or Body Volume Index with the caveat that the child's volume is $\pi$ times the height times the abdominal radius squared times, which is relatively constant relative to height. The notion for this metric is that weight density, or weight per unit volume, is the child's weight divided by a cylindrical volume that is equivalent to the body's volume. This assumes that the child's volume can be mapped into a cylinder. The volume of the cylinder is the child's height times the area of a circle with radius, $r$. Here $r$ is an average radius, which takes into consideration the variation in the width of the child's shoulder, chest, abdomen, and head. We expect the radius to have less variation than the height so that it is not needed in the density calculation. Thus, the weight-density is simply proportional to the ratio of weight to height of a child.

This may be a reasonable metric for infants since the development of the abdominal muscles of an infant and changes in girth are much slower than the increase in the infant's height [20]. We expect the radius to have less variation than the height so it is not needed in the density calculation. Thus, the weightdensity is simply proportional to the ratio of weight to height of a child. The Center for Disease Control (CDC) publishes a growth chart for boys and girls in the United States from birth to 36 months entitled "Weight-from Length" percentiles [22]. This chart has a constant slope for each percentile, which is the WOH metric considered here.

\section{Longitudinal Decision Analysis (LDA) for Predicting Obesity}

In this section we develop a longitudinal decision analysis (LDA) method, which is similar to an individuals type control chart analysis about a trendline, using the normal cohort as the control group of individuals to assess whether or not a child at any time measured in dsb is potentially a candidate to be obese by five years of age. Figures 1 and 2 illustrate the relationships between BMI and $\mathrm{WOH}$ versus dsb for the normal and obese cohorts. The two sets of figures suggest that WOH characterizes the normal cohort better than BMI based on the spread of the profiles for the normal children in this study. But the data for both the normal and obese cohorts indicate that the $\mathrm{WOH}$ ratio is an increasing function of dsb, with the normal cohort increasing linearly starting at about one year. 


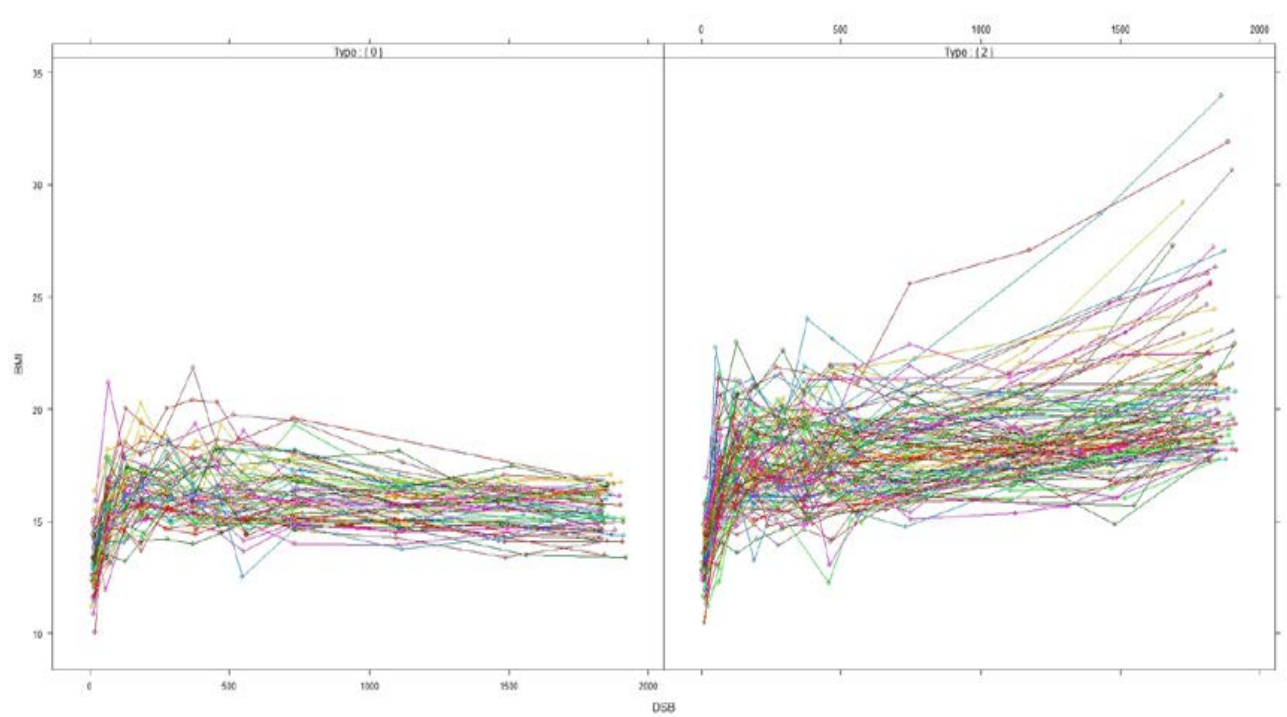

Figure 1: Plot for BMI versus dsb (Left panel represents the "normal" group; Right panel represents the "obese" group)

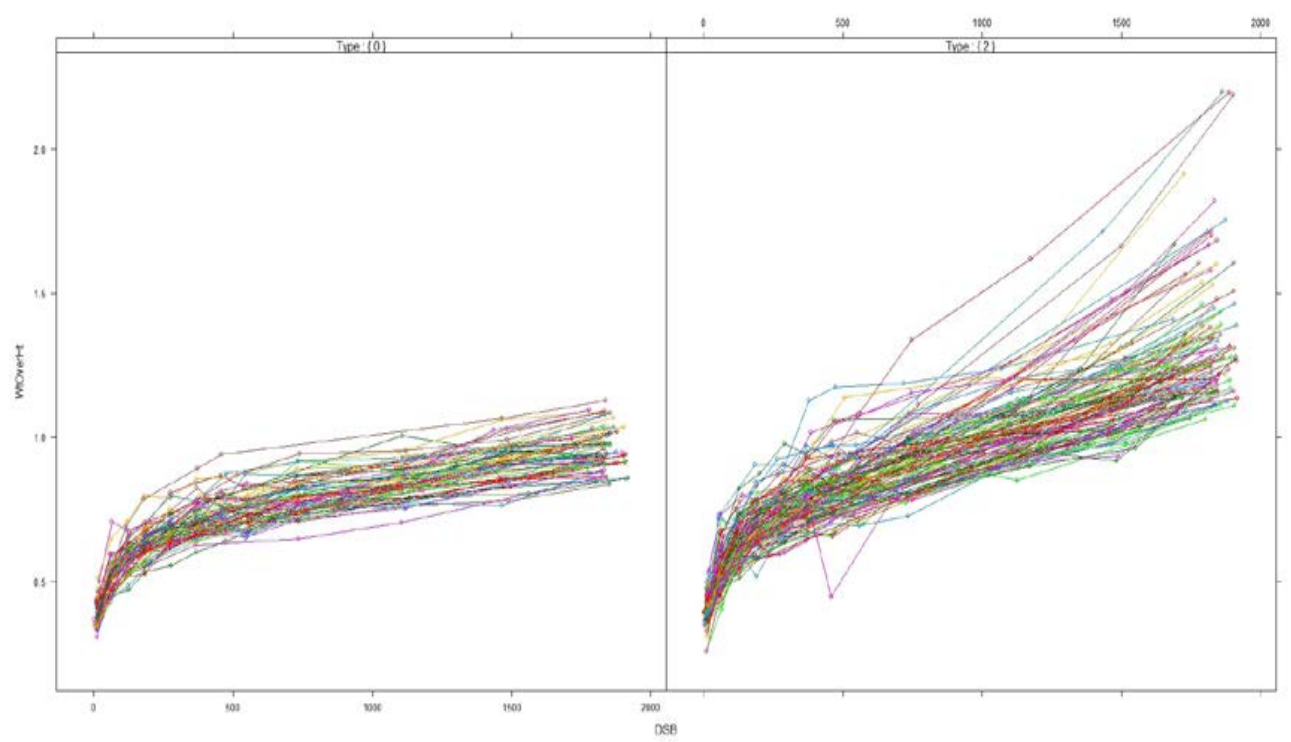

Figure 2: Plot for WOH versus dsb (Left panel represents the "normal" group; Right panel represents the "obese" group)

LDA is generated by developing a linear regression model for $\mathrm{WOH}$ as a function of the scaled, exponential variable, $\exp (-\mathrm{dsb} / 454)$, using ordinary least squares. In particular, the growth curve model in the following equation was obtained using data from the normal cohort. 
$\mathrm{WOH}($ predicted $)=0.929-0.494 \exp (-\mathrm{dsb} / 454)$.

The $R^{2}$ for this model generated from the data from the normal cohort, which is the sum of square differences between the fitted values and the mean $\mathrm{WOH}$ divided by the sum of square differences between the $\mathrm{WOH}$ values and the mean $\mathrm{WOH}$, indicates that $85.8 \%$ of the variation in $\mathrm{WOH}$ is explained. The square root of the mean square error (RMSE), which is the square root of the sum of squared residuals divided by $(61-2)$, is 0.066 .

The standard error of estimate (se) can determined for each of the 61 members of the normal cohort from the expression,

$$
\operatorname{RMSE} * \sqrt{x_{i}\left(X^{t} X\right)^{-1} x_{i}^{t}},
$$

where $X$ is the $61 \times 2$ matrix with $i$-th row defined as $x_{i}=\left(1, \exp \left(-\mathrm{dsb}_{i} / 454\right)\right.$. With the standard errors computed for each of the 61 children in the normal cohort, a linear regression equation was fitted to these standard errors as shown in the equation below.

$$
\text { se }=0.078-0.84 * 10^{-6} * \mathrm{dsb} .
$$

From this model an upper 95\% confidence interval profile for the WOH versus dsb can be generated using the model above for the standard error (se) of prediction.

$$
\mathrm{UDL}(k)_{\mathrm{WOH}}=\mathrm{WOH}(\text { predicted })+k * \text { se. }
$$

The upper $k$-sigma decision limit, $\mathrm{UDL}(k)_{\mathrm{WOH}}$, for the normal cohort is a function of dsb. Two $k$-sigma values were considered, $k$ equal to 2 and $k$ equal to 3 , which are similar to the 3-sigma Shewhart limit [23] and the 2-sigma warning limit defined by Ott [24].

\section{4. $\mathrm{UDL}(k)_{\mathrm{WOH}}$ as A Predictor of Obesity}

Table 3 summarizes the performance of the $\operatorname{UDL}(k)_{\mathrm{WOH}}$ with $k$ equal to 2 and 3 as a limit to predict obesity for an individual for the normal and obese cohorts at two years of age. Performance is measured in terms of sensitivity (percentage of normal weight who are predicted to be normal weight), specificity (percentage of obese who are predicted to be obese), positive predictive value (PPV - percentage of predicted normal weight who are actually normal weight) and negative predictive value (NPV - percentage of predicted obese who are actually obese).

For children up to two years of age, the UDL $(2)_{\mathrm{WOH}}$ profile shown in Figure 3 can be used to identify any child by age two who is at risk of becoming obese 
Table 3: WOH performance matrix based on $\operatorname{UDL}(k)_{\mathrm{WOH}}$ at 2 -yoa

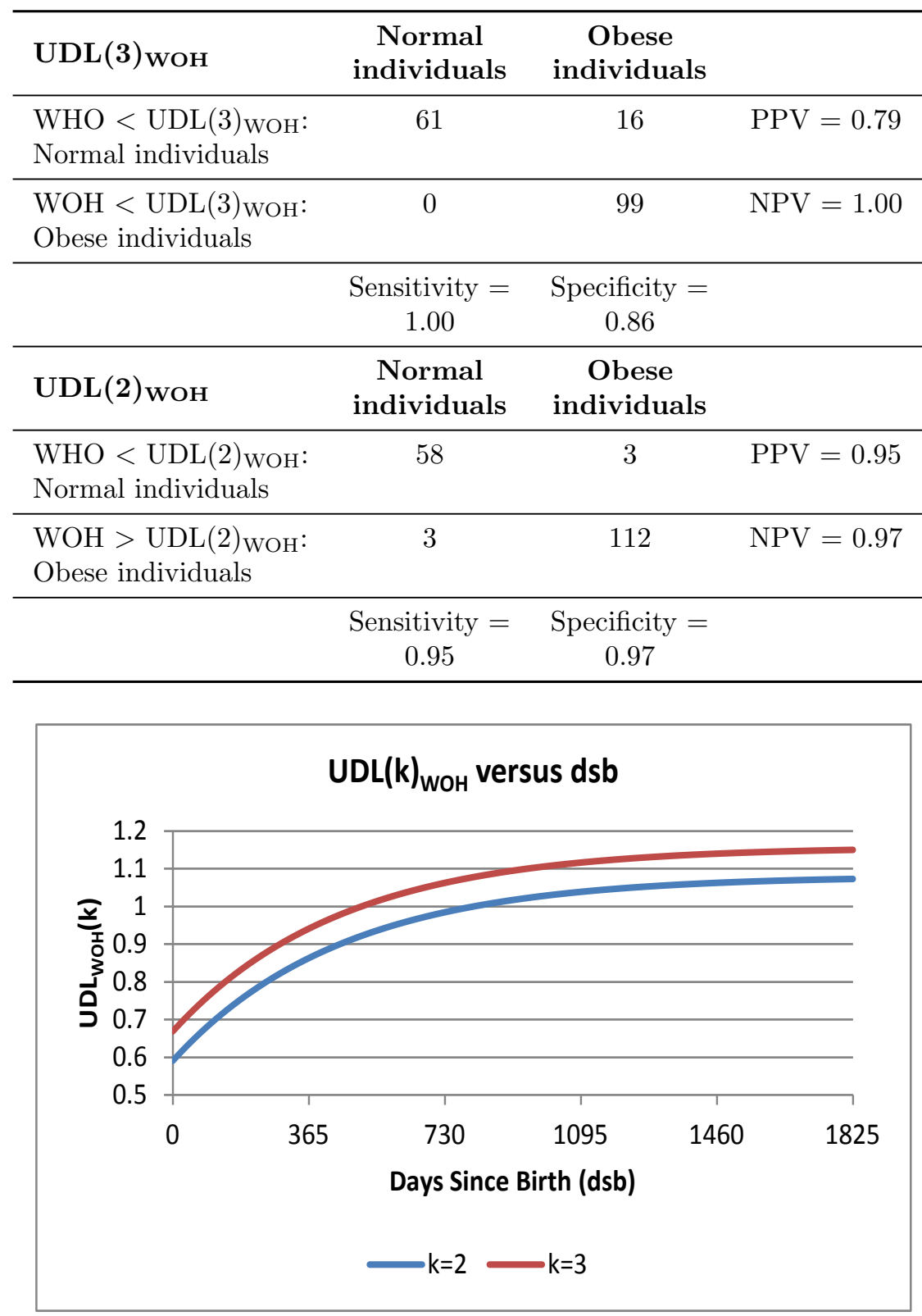

Figure 3: UDL $(k)_{\mathrm{WOH}}$ profiles

by the age of five. The distribution of the first dsb when obesity is predicted using the criterion: $\mathrm{WOH}>\mathrm{UDL}(2)_{\mathrm{WOH}}$ is shown in Figure 4 . It is noteworthy that $75 \%$ of the obese cohort is predicted to be obese at 64 dsb and $84 \%$ at 182 dsb. 


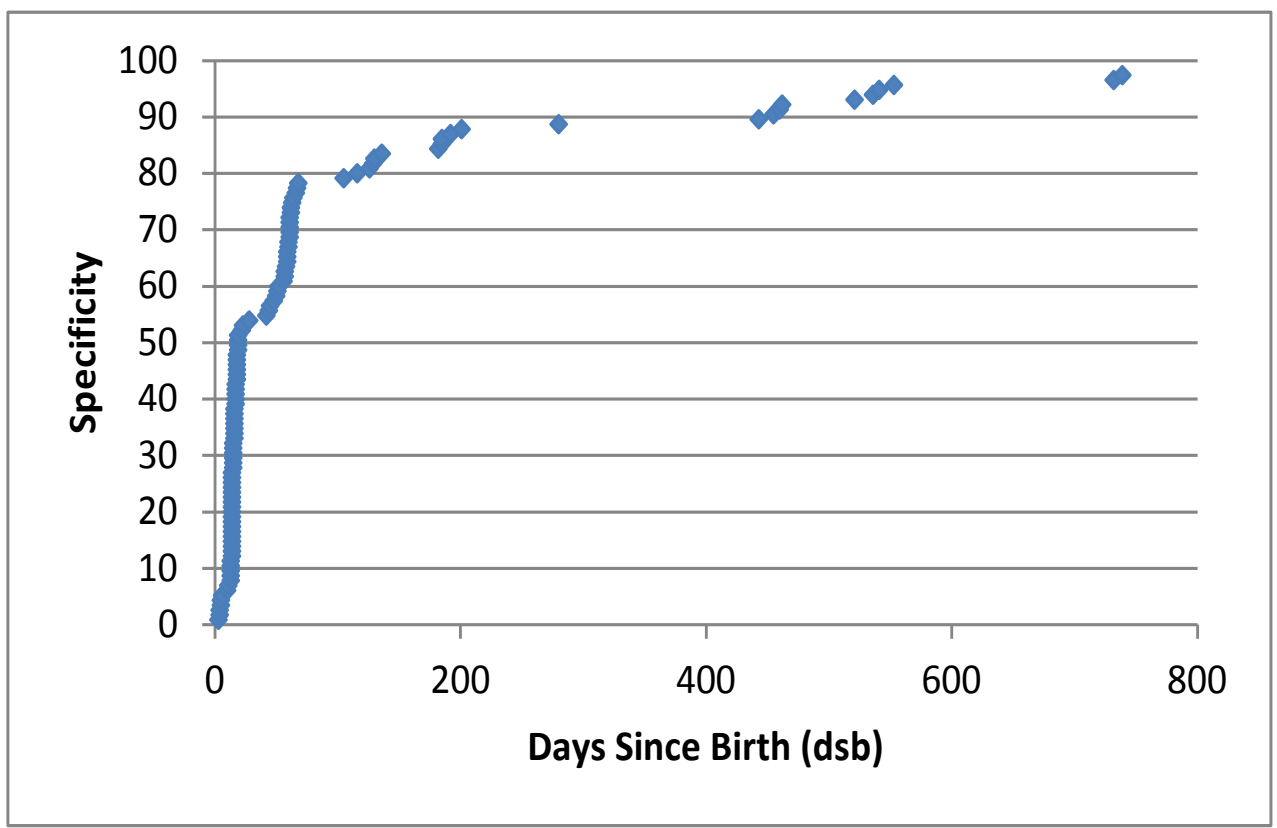

Figure 4: Specificity using the dsb when obesity is first predicted with $\mathrm{UDL}(2)_{\mathrm{WOH}}$

\section{Analysis and Effectiveness of BMI versus dsb at Predicting Obesity}

Figure 1 illustrates the relationship between BMI and days-since-birth (dsb) for the two extreme cohorts, normal and obese children. The normal cohort children shown in Figure 1 appear to represent a stable process as defined by Shewhart [23]. The concept of a Shewhart control chart for analyzing time ordered or longitudinal data states that a process is stable or under control if all the data are within the upper and lower control limits (UCL and LCL) defined as follows:

$$
\mathrm{LCL}=\bar{Y}-3(\text { Median MR/0.954), } \mathrm{UCL}=\bar{Y}+3(\text { Median MR/0.954). }
$$

Here $\bar{Y}$ is the average BMI. These control limits are computed for individual data using the median moving range (MR) or the absolute successive differences of BMI values from one hospital visit to the next divided by 0.954 as the standard deviation. Suppose the normal cohort of children is treated as the control group. For this group of 61 children, the average of the upper control limits for each child serves as a limit to detect instability in the BMI before two years of age. If a single point is above the upper control limit by the age of two, then the child's BMI viewed as a system rather than a single data point is out of control, and the 
decision is that the child could become obese by five years of age if there is no intervention to change the system.

Ellis Ott [24] suggests the following upper and lower warning limits (UWL and LWL) to identify opportunities to learn more about the process or to adjust it. These limits increase the risk of making a type one error, but reduce the risk of a type two error.

$$
\mathrm{LWL}=\bar{Y}-2(\text { Median MR/0.954), } \quad \mathrm{UWL}=\bar{Y}+2(\text { Median MR/0.954) } .
$$

This less conservative approach can be used to identify children at the age of two who could become obese by the age of five. If by age two a single data point is above the upper warning limit, then the parents should be warned by the physician that the child could become obese by the age of five.

As in the analysis of $\mathrm{WOH}$, the normal cohort of children, i.e., those known to have normal weight by the CDC standards at age five, were considered the control group. The average UCL and UWL of the 61 children in this group are 18.45 and 17.54, respectively. Applying these limits to the normal and obese groups, Table 4 was generated.

Table 4: BMI performance matrix based on UCL and UWL at 2-yoa

\begin{tabular}{|c|c|c|c|}
\hline $\mathbf{U C L}_{\mathrm{BMI}}$ & $\begin{array}{c}\text { Normal } \\
\text { individuals }\end{array}$ & $\begin{array}{c}\text { Obese } \\
\text { individuals }\end{array}$ & \\
\hline $\begin{array}{l}\mathrm{BMI}<\mathrm{UCL}_{\mathrm{BMI}}: \\
\text { Normal individuals }\end{array}$ & 47 & 48 & $\mathrm{PPV}=0.49$ \\
\hline \multirow[t]{2}{*}{$\begin{array}{l}\text { BMI > UCL } \mathrm{UMI}_{\mathrm{BMI}} \\
\text { Obese individuals }\end{array}$} & 14 & 67 & $\mathrm{NPV}=0.83$ \\
\hline & $\begin{array}{c}\text { Sensitivity }= \\
0.77\end{array}$ & $\begin{array}{c}\text { Specificity }= \\
0.58\end{array}$ & \\
\hline $\mathbf{U W L}_{\mathrm{BMI}}$ & $\begin{array}{c}\text { Normal } \\
\text { individuals }\end{array}$ & $\begin{array}{c}\text { Obese } \\
\text { individuals }\end{array}$ & \\
\hline $\begin{array}{l}\mathrm{BMI}<\mathrm{UWL}_{\mathrm{BMI}}: \\
\text { Normal individuals }\end{array}$ & 35 & 25 & $\mathrm{PPV}=0.58$ \\
\hline \multirow[t]{2}{*}{$\begin{array}{l}\text { BMI > UWL } \mathrm{UMI}_{\mathrm{BMI}}: \\
\text { Obese individuals }\end{array}$} & 26 & 90 & $\mathrm{NPV}=0.77$ \\
\hline & $\begin{array}{c}\text { Sensitivity }= \\
0.57\end{array}$ & $\begin{array}{c}\text { Specificity }= \\
0.78\end{array}$ & \\
\hline
\end{tabular}

The sensitivity, specificity, positive predictive value (PPV) and negative predictive value (NPV) for decision making using BMI by the age of two based on 
the UCL and UWL are indicated in these tables. The results shown in Table 4 indicate that both the control chart limits and warning limits for BMI do not have sufficient Sensitivity and PPV. Thus the Shewhart control chart applied to BMI measurements up to the age of two should not be considered as a means to intervene on behalf of a child by counseling parents or guardians relative to a child's weight development. The WOH metric is superior to BMI for predicting obesity in children at age five before two years of age.

\section{Conclusion}

We are proposing the LDA chart in Figure 3 based on the weight-height ratio, $\mathrm{WOH}$, rather than $\mathrm{BMI}$ for predicting obesity by age five of an individual before their second birthday if there is no intervention by the child's physician to change the behavior pattern of the family or guardian. In particular, the UDL $(2)_{\mathrm{WOH}}$ profile has a $95 \%$ sensitivity and PPV and $97 \%$ specificity and NPV for predicting obesity at age five by two years of age without such intervention. Moreover, $75 \%$ of the individual children in the obese cohort were identified as obese by approximately two months and $84 \%$ by approximately three months.

This is a retrospective study, based on a total sample size of 176 child-mother dyads for which there were a minimum of 8 well-child visits from birth through five years of age. Three points should be emphasized based on the methods proposed for using the LDA chart. This method requires data on weight and height of the child at approximately one week, 2, 4, 6, 9, 12, 15, 18 month, and 2 year well-child visits. Without records of these data, the physician cannot predict whether or not the child will be obese at age five. Second, although demographic data were not available for inclusion in the analysis, based on the 2000 census of the 9-county HMO service area from which our sample of mother-child dyads were obtained, $74 \%$ of the people are Caucasian, $22 \%$ are African-American, and $2 \%$ are Asian. Our assumption is that a similar racial profile is reflected in the sample of 176 mother-child dyads as the corresponding population in the HMO service area. Thus, the accuracy of our methods for predicting obesity at age 5 based on the WHO profile at or before age two may not be as good for demographic distributions that are significantly different from the population from which our samples were selected. In addition it should be pointed out that although our analyses were able to identify infants who were morbidity obese, the prevalence of morbid obesity may actually be more than expected in demographic distributions different from that of our study. Finally, it should be pointed out that the data were not stratified based on gender, partly because the cohort sizes were small. Gender should be included in future obesity studies of individuals when larger data sets are available for analysis. 


\section{References}

[1] Popkin, B. M., Kim, S., Rusev, E. R., Du, S. and Zizza, C. (2006). Measuring the full economic costs of diet, physical activity and obesity-related chronic diseases. Obesity Reviews 7, 271-293.

[2] Centers for Disease Control and Prevention. (2010). Preventing obesity and chronic diseases through good nutrition and physical activity. Available at http://www.cdc.gov/nccdphp/pe_factsheets/pe_pa.htm.

[3] Short, K. R., Blackett, P. R., Gardner, A. W. and Copeland, K, C. (2009). Vascular health in children and adolescents: effects of obesity and diabetes. Vascular Health and Risk Management 5, 973-990.

[4] Sangun, Ö., Dundar, B., Köşker, M., Pirgon, Ö. and Dündar, N. (2011). Prevalence of metabolic syndrome in obese children and adolescents using three different criteria and evaluation of risk factors. Journal of Clinical Research in Pediatric Endocrinology 3, 70-76.

[5] Falkner, B. (2008). Children and adolescents with obesity-associated high blood pressure. Journal of the American Society of Hypertension 2, 267274 .

[6] Freedman, D. S., Mei, Z., Srinivasan, S. R., Berenson, G. S. and Dietz, W. H. (2007). Cardiovascular risk factors and excess adiposity among overweight children and adolescents: the Bogalusa Heart Study. Journal of Pediatrics 150, 12-17.e2.

[7] van Vliet, M., Heymans, M. W., von Rosenstiel, I. A., Brandjes, D. P., Beijnen, J. H. and Diamant, M. (2011). Cardiometabolic risk variables in overweight and obese children: a worldwide comparison. Cardiovascular Diabetology 10, 106.

[8] National Institutes of Health. (2007). Clinical guidelines on the identification, evaluation, and treatment of overweight and obesity in adults: the evidence report. Bethesda, National Institutes of Health, Maryland, U.S. Department of Health and Human Services.

[9] Han, J. C., Lawlor, D. A. and Kimm, S. Y. (2010). Childhood obesity. Lancet 375, 1737-1748.

[10] Sutherland, E. R. (2008). Obesity and asthma. Immunology and Allergy Clinics of North America 28, 589-602, ix. 
[11] Whitlock, E. P., Williams, S. B., Gold, R., Smith, P. R. and Shipman, S. A. (2005). Screening and interventions for childhood overweight: a summary of evidence for the US Preventive Services Task Force. Pediatrics 116, $125-144$.

[12] Cornette, R. (2008). The emotional impact of obesity on children. Worldviews on Evidence-based Nursing 5, 136-141.

[13] Dietz, W. H. (1998). Health consequences of obesity in youth: childhood predictors of adult disease. Pediatrics 101, 518-525.

[14] Swartz, M. B. and Puhl, R. (2003). Childhood obesity: a societal problem to solve. Obesity Reviews 4, 57-71.

[15] Cole, T. J., Bellizzi, M. C., Flegal, K. M. and Dietz, W. H. (2000). Establishing a standard definition for child overweight and obesity worldwide: international survey. BMJ 320, 1240-1243.

[16] Power, C., Lake, J. K. and Cole, T. J. (1997). Body mass index and height from childhood to adulthood in the 1958 British born cohort. American Journal of Clinical Nutrition 66, 1094-1101.

[17] Power, C., Lake, J. K. and Cole, T. J. (1997). Measurement and longterm health risks of child and adolescent fatness. International Journal of Obesity 21, 507-526.

[18] Ludington-Hoe, S. M., Gittner, L. S. and Haller, H. S. (2012). A pilot study: Does obesity begin in the first year of life? To appear in Clinical Pediatrics.

[19] Lampl, M., Gotsch, F., Kusanovic, J. P., Gomez, R., Nine, J. K., Frongillo, E. A. and Romero, R. (2010). Sex differences in fetal growth responses to maternal height and weight. American Journal of Human Biology 22, 431-443.

[20] Keys, A., Fidanza, F., Karvonen, M. J., Kimura, N. and Taylor, H. L. (1972). Indices of relative weight and obesity. Journal of Chronic Diseases 22, 329-343.

[21] Burdi, A. R., Huelke, D. F., Snyder, R. G. and Lowrey, G. H. (1969). Infants and children in the adult world of automobile safety design: pediatric and anatomical considerations for design of child restraints. Journal of Biomechanics 2, 267-280. 
[22] Centers for Disease Control and Prevention. (2002). Developed by the National Center for Health Statistics in collaboration with the National Center for Chronic Disease Prevention and Health Promotion. Available at http://www.cdc.gov/growthcharts.

[23] Shewhart, W. A. (1938). Application of statistical method in mass production. In Proceedings of the Industrial Statistics Conference Held at Massachusetts Institute of Technology, September 8-9. Pitman Publishing Corporation, New York.

[24] Ott, E. R. (1975). Process Quality Control. McGraw-Hill Book Company, New York.

Received September 6, 2012; accepted December 14, 2012.

\author{
Harold S. Haller \\ Director, Case Statistical Consulting Center \\ Department of Statistics \\ Case Western Reserve University \\ 10900 Euclid Avenue, Cleveland, OH 44106-7054, USA \\ Hsh4@case.edu \\ Tanujit Dey \\ Department of Mathematics \\ College of William \& Mary \\ Williamsburg, VA 23185, USA \\ tdey@wm.edu \\ Lisaann S. Gittner \\ Tennessee State University \\ College of Public Service and Urban Affairs \\ Department of Public Administration \\ 330-10th Ave. North, Nashville, TN 37203, USA \\ lgittner@tnstate.edu \\ Susan M. Ludington-Hoe \\ FP Bolton School of Nursing \\ Case Western Reserve University \\ 10900 Euclid Avenue, Cleveland, OH 44106, USA \\ Sml15@case.edu
}

